\title{
ON WEIGHTED INTEGRAL EQUATIONS WITH NEGATIVE EXPONENTS
}

\section{LiLI HUANG}

Abstract. This paper is concerned with the integral equation

$$
u(x)=\int_{R^{n}}|x-y|^{p} k(y) u^{q}(y) d y,
$$

where $n \geqslant 1, p \neq 0, q<0$, and the weighted function $k(x)$ is smooth. This equation comes from the prescribing curvature problems. In addition, it is related to the best functions of the reversed Hardy-Littlewood-Sobolev inequality. We consider the existence and the estimates of increasing rates of the positive entire solutions in the case that $k(x)$ is bounded and unbounded respectively.

Mathematics subject classification (2010): 45E10, 45G15, 45M05, 45M20.

Keywords and phrases: Integral equation, negative exponent, existence of entire solutions, increasing rates.

\section{REFERENCES}

[1] J. Byeon, Z. WANG, On the Henon equation: Asymptotic profile of ground states, II, J. Differential Equations, 216 (2005), 78-108.

[2] M. CAlanchi, B. RUF, Radial and non radial solutions for Hardy-Henon type elliptic systems, Calc. Var. Partial Differential Equations, 38 (2010), 111-133.

[3] W. Chen, C. LI, B. OU, Qualitative properties of solutions for an integral equation, Discrete Contin. Dyn. Syst., 12 (2005), 347-354.

[4] W. Chen, C. LI, B. OU, Classification of solutions for an integral equation, Comm. Pure Appl. Math., 59 (2006), 330-343.

[5] J. Dou, M. ZHU, Reversed Hardy-Littlewood-Sobolev inequality, Internat. Math. Res. Notices, 2014 (2014), rnu241, 31 pages.

[6] J. KAZDAN, F. WARNER, Existence and conformal deformation of metrics with prescribing Gaussian and scalar curvature, Ann. of Math., 101 (1975), 317-331.

[7] Y. LEI, Asymptotic properties of positive solutions of the Hardy-Sobolev type equations, J. Differential Equations, 254 (2013) 1774-1799.

[8] Y. LEI, On the integral systems with negative exponents, Discrete Contin. Dyn. Syst., 35 (2015), $1039-1057$.

[9] Y.-Y. LI, Remark on some conformally invariant integral equations: the method of moving spheres, J. Eur. Math. Soc., 6 (2004), 153-180.

[10] Y. LI, W.-M. NI, On conformal scalar curvature equations in $R^{n}$, Duke Math. J., 57 (1988), 895-924.

[11] G. LU, J. ZHU, Symmetry and regularity of extremals of an integral equation related to the HardySobolev inequality, Calc. Var. Partial Differential Equations, 42 (2011), 563-577.

[12] S. SUn, Y. LEI, Fast decay estimates for integrable solutions of the Lane-Emden type integral systems involving the Wolff potentials, J. Funct. Anal., 263 (2012), 3857-3882.

[13] X. XU, Uniqueness theorem for integral equations and its application, J. Funct. Anal., 247 (2007), $95-109$.

[14] W. ZIEMER, Weakly Differentiable Functions, Grad. Texts in Math., vol. 120, Springer-Verlag, Berlin, 1989. 